\title{
A Novel CP Horn Antenna with Switchable Polarization by Single Port Feeding
}

\author{
Yangzhen Huang, Junping Geng, Xianling Liang, Ronghong Jin, and Xudong Bai \\ Department of Electronic Engineering, Shanghai Jiao Tong University, No. 800 Dongchuan Road, Shanghai 200240, China \\ Correspondence should be addressed to Junping Geng; gengjunp@sjtu.edu.cn
}

Received 13 December 2014; Accepted 6 February 2015

Academic Editor: Giuseppe Mazzarella

Copyright (c) 2015 Yangzhen Huang et al. This is an open access article distributed under the Creative Commons Attribution License, which permits unrestricted use, distribution, and reproduction in any medium, provided the original work is properly cited.

\begin{abstract}
A novel CP horn antenna with switchable polarization by single port feeding is presented. The antenna is composed of a coaxialwaveguide converter and a balance-cavity, a horn, a square-to-circular waveguide transition, and a stepped-septum polarizer, which simplify the feeding and can work on the left-hand or right-hand circular polarizations by manual exchanging the mounting position of the coaxial-waveguide converter and the balance-cavity. The antenna achieves an impedance bandwidth (3.88 5.12 GHz) of $27.5 \%$ and a $3 \mathrm{~dB}-\mathrm{AR}$ (Axial Ratio) bandwidth of $18.6 \%$, with the $3 \mathrm{~dB}-\mathrm{AR}$ beamwidth of about $149^{\circ}$, and the antenna gain is over $13.3 \mathrm{~dB}$ in the available band.
\end{abstract}

\section{Introduction}

Circular polarized (CP) antenna has been considered as the key component of the high data rate communication system for antidepolarization [1], easy matching polarization, and suppressing the interference of Faraday rotation effect from the water droplet or ionosphere in channel [2]. In addition, $\mathrm{CP}$ antenna with switchable polarization may be more attractive in order to increase the efficient utilization of the electromagnetic spectrum and the link capacity [3]. The usual method to obtain switchable polarization is utilizing the properties of PIN diode in the microstrip antennas $[4,5]$, but these antennas cannot match the requirements of longer range wireless communication and horn antenna maybe a better choice because of its high efficiency and high gain with great power capacity. In [6], a horn antenna is presented to realize the broadband polarization splitting, but the dual-port feeding configuration may increase the system complexity and the processing cost of antenna, which is inconvenient in miniaturized system. Consequently, a novel CP horn antenna with switchable polarization by single port feeding is introduced and carefully examined.

In this paper, we present a novel CP horn antenna with single-feeding port and switchable polarization, which is attractive in microwave communication, especially in millimeter wave applications compared with other frequency bands. In this study, constrained by various conditions, such as test equipment and machining accuracy, the proposed antenna is machined and measured in C-band to confirm the design idea, which can be easily extrapolated to other bands. The antenna is fed by a coaxial-waveguide converter along with a balance-cavity which keeps a wideband impedance matching and achieves the left-hand or right-hand circular polarizations by altering the connecting method between the polarizer and the single-feeding system (including the converter and the balance-cavity). The optimized antenna can achieve an available impedance bandwidth $(3.88 \sim 5.12 \mathrm{GHz})$ of $27.5 \%$ and a $3 \mathrm{~dB}$ AR bandwidth of $18.6 \%$. The antenna gain is over $13.3 \mathrm{~dB}$ in the operation band with the $3 \mathrm{~dB}$-AR beam width over $149^{\circ}$.

\section{Antenna Geometry and Design}

The proposed CP antenna is schematically represented in Figure 1, which includes a horn, a square-to-circular waveguide transition, a stepped-septum polarizer, and a singlefeeding system (including a coaxial-waveguide converter and a balance-cavity). The horn is composed of two sections: a conical horn section and a loaded circular waveguide section, which can obtain a compact geometry and high aperture efficiency [7]. The square-to-circular waveguide transition, 


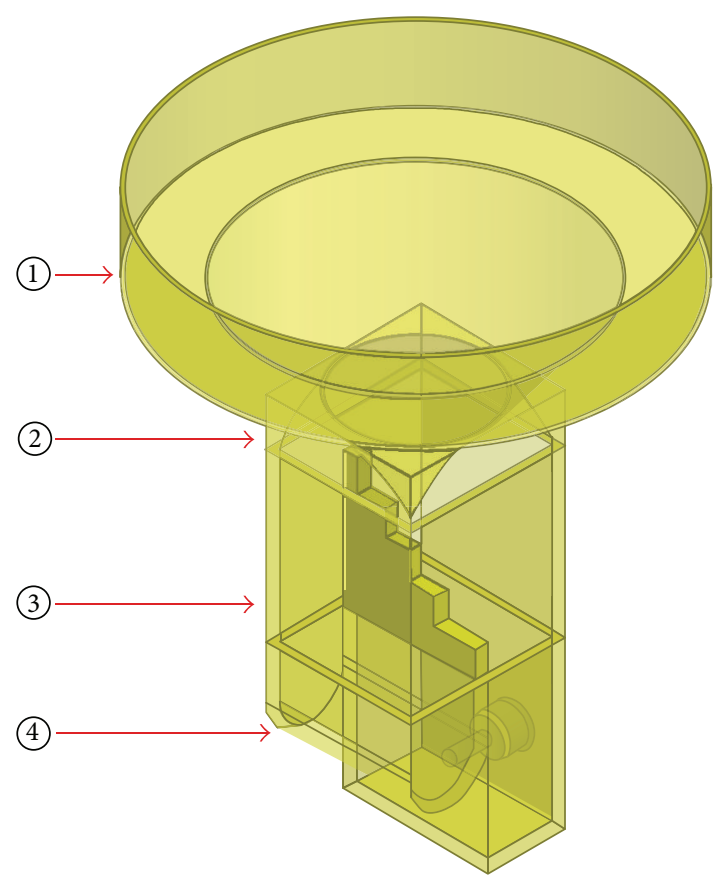

(a)
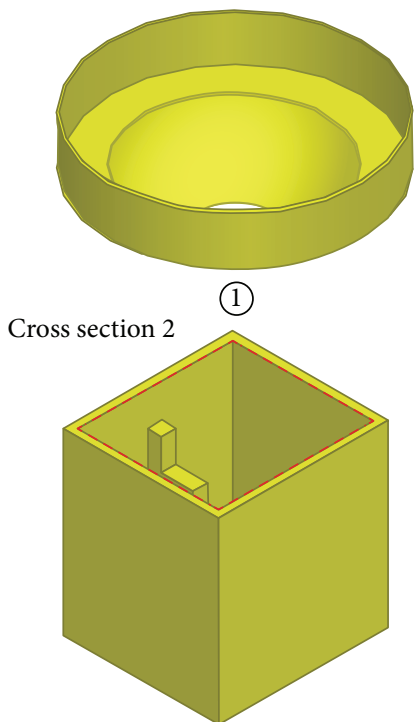

(3)

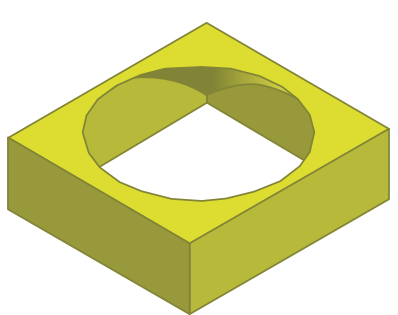

(2) Cross section 1

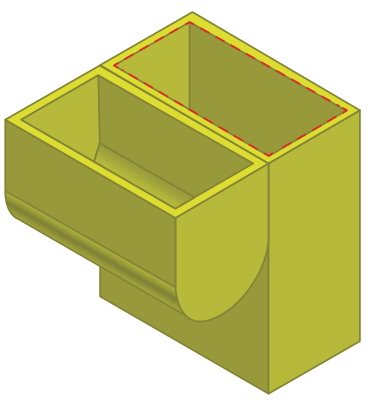

(4)

(b)

FIGURE 1: Geometry of proposed antenna (a). Integrated structure; (b). (1) Horn, (2) transition (3) polarizer, and (4) Single feeding.

achieving a smooth transition between two modes of electromagnetic wave, is introduced to connect the horn and the stepped-septum polarizer [8].

The polarizer is a three-port waveguide device which is divided into two parts through a thin conductive septum, leaving us with two identical input rectangular waveguide ports and a single output square waveguide port [9]. By exciting one input port and connecting impedance matching on the other input port simultaneously, the circular polarization wave can be produced at the output square waveguide, which is a combination of $\mathrm{TE}_{10}$ and $\mathrm{TE}_{01}$ modes [10]. In this study, one port is replaced by the balance-cavity, which plays the role of energy reflector and phase compensator; the other port is excited by the coaxial-waveguide converter. The electromagnetic wave excitation coupled to the other part of the polarizer waveguide through the complicated coupling mechanism in the septum region and reflected by the balance-cavity [11]. A proper phase relationship between the reflected electromagnetic wave and the initial excitation can be obtained via the cavity structure adjustment. Combined design of the cavity and the stepped-septum is carried out to achieve a good performance in terms of axial ratio and impedance matching.

The realization of the polarization switching is based on the feature that the stepped-septum polarizer can excite high-purity LHCP or RHCP [12]. When one input port is excited, the LHCP is produced; when the other input port is excited, the RHCP is produced. Consequently, simple mechanical rotation of the single-feeding system can realize the switchable polarization. The properties of the two types of polarization modes are similar, which is determined by the symmetry prosperities of the structure. The study aims to simplify antenna construction and that this is indeed the case has been confirmed experimentally and the results are presented in the following section.

2.1. The Design Process of Balance-Cavity. As shown in Figure 1(a), the simulation and optimization is firstly based on the LHCP mounting-method, and the balance-cavity and the coaxial-waveguide converter are connected with the two input ports of polarizer, respectively. As illustrated in Figure 1(b), cross section 1 of input rectangular waveguide has the side length $40.386 \mathrm{~mm} \times 20.193 \mathrm{~mm}$ and the cutoff frequency of fundamental mode $\left(\mathrm{TE}_{10}\right)$ and higher-order modes $\left(\mathrm{TE}_{01} / \mathrm{TE}_{20} / \mathrm{TE}_{11} / \mathrm{TM}_{11}\right)$ are $3.71,7.43,7.43,8.31$, and $8.31 \mathrm{GHz}$, respectively, which can realize the single-mode transmission $\left(\mathrm{TE}_{10}\right)$ of electromagnetic wave in coaxial-waveguide converter and thus generate $\mathrm{TE}_{10}$ excitation signal. Cross section 2 of output square waveguide is shown in Figure 1(b), which has the side length $42.386 \mathrm{~mm} \times 40.386 \mathrm{~mm}$ and the cutoff frequency of fundamental mode $\left(\mathrm{TE}_{10}\right)$ and higher-order modes $\left(\mathrm{TE}_{01} / \mathrm{TE}_{11} / \mathrm{TM}_{11} / \mathrm{TE}_{20}\right)$ are 3.54, 3.71, 5.13, 5.13, and $7.08 \mathrm{GHz}$, respectively, which can achieve the dual-modes transmission $\left(\mathrm{TE}_{10} / \mathrm{TE}_{01}\right)$ of electromagnetic wave and thus generate the circularly polarized output.

On this basis, the design of balance-cavity is carried out and it is expected that best performance in terms of axial ratio and impedance matching will result by choosing 


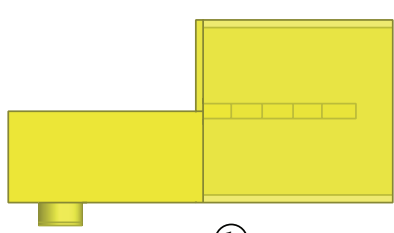

(1)

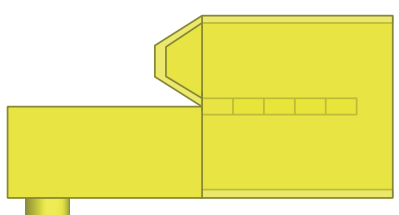

(3)

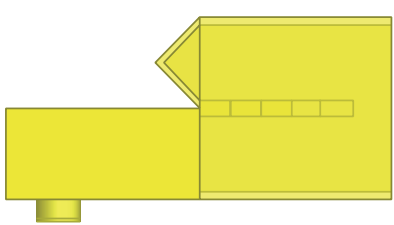

(2)

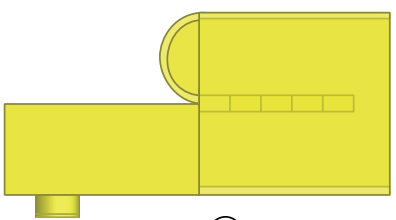

(4)

(a)

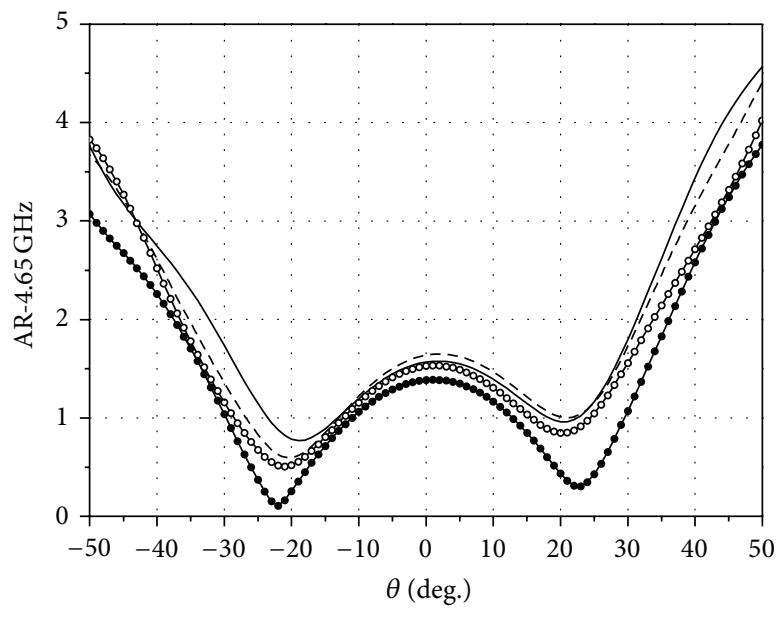

- (A) Rectangle

--- (B) Triangle $\multimap$ (C) Trapezium

(D) Semicircle

(b)

FIgURE 2: (a) Four shapes: (1) rectangle, (2) triangle, (3) trapezium, and (4) semicircle; (b) AR at $4.65 \mathrm{GHz}$.

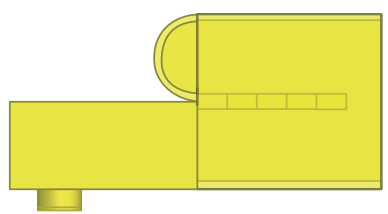

(1)

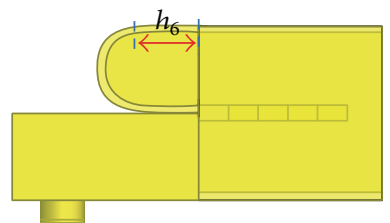

(3)

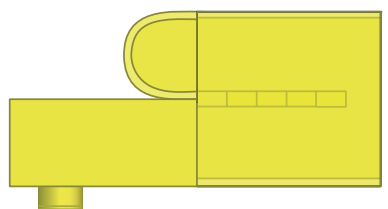

(2)

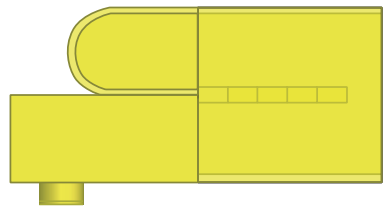

(4)

(a)

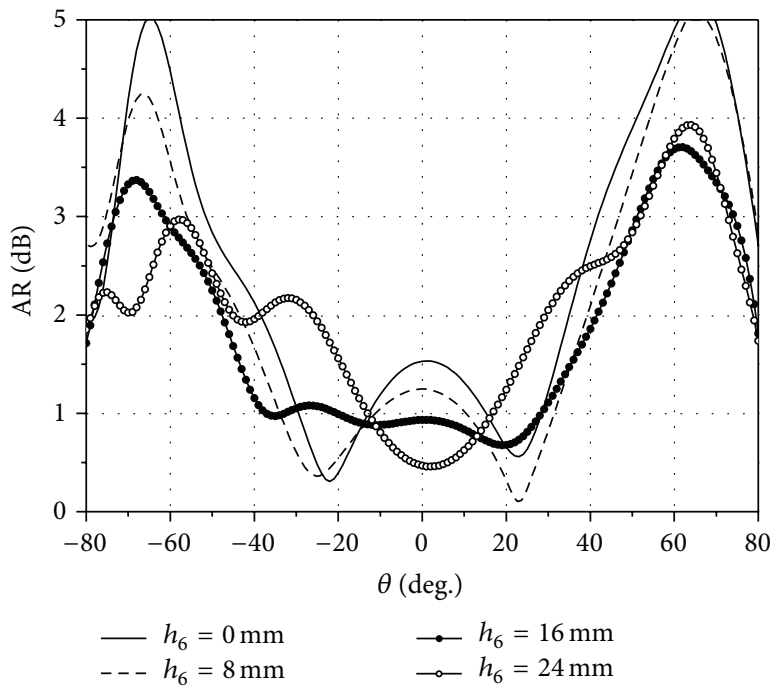

(b)

Figure 3: The effects of $h_{6}$ on antenna: (a) four lengths, (b) AR at $4.65 \mathrm{GHz}$.

appropriate cavity configuration. As shown in Figure 2, the shape of the inner reflective surface of the balance-cavity is an important indicator which affects the $3 \mathrm{~dB}-\mathrm{AR}$ (axial ratio) bandwidth and the circularly polarized wave beamwidth. To better understand the effects, several types of shape (rectangle, triangle, trapezium, and semicircle) are modelled and simulated in HFSS, and antenna performance for these cases is illustrated in Figure 2(b) and summed up in Table 1. According to the simulation results, the semicircle has the best performance in terms of $3 \mathrm{~dB}-\mathrm{AR}$ (axial ratio) bandwidth and the circularly polarized wave beamwidth. As shown in Figure 3, the effects of $h_{6}$ (the length of balance-cavity) are simulated and optimized in HFSS. It is observed that the parameter $h_{6}$ has a relatively significant influence on
TABLE 1: Four shapes of inner reflective surface in Figure 2.

\begin{tabular}{lcccc}
\hline Type & Rectangle & Triangle & Trapezium & Semicircle \\
\hline 3 dB-AR bandwidth & $10.3 \%$ & $11.8 \%$ & $14 \%$ & $15.8 \%$ \\
\hline $\begin{array}{l}3 \mathrm{~dB}-\mathrm{AR} \text { beamwidth } \\
4.65 \mathrm{GHz}\end{array}$ & $82^{\circ}$ & $83^{\circ}$ & $86^{\circ}$ & $96^{\circ}$ \\
\hline
\end{tabular}

the AR performance-curve along the theta coordinate axis. The length $h_{6}$ is determined to be $24 \mathrm{~mm}, 16 \mathrm{~mm}, 8 \mathrm{~mm}$, and $0 \mathrm{~mm}$, respectively, to achieve best performance and the simulated $3 \mathrm{~dB}-\mathrm{AR}$ beamwidth at $4.65 \mathrm{GHz}$ is $120^{\circ}$ when $h_{6}=$ $16 \mathrm{~mm}$. 


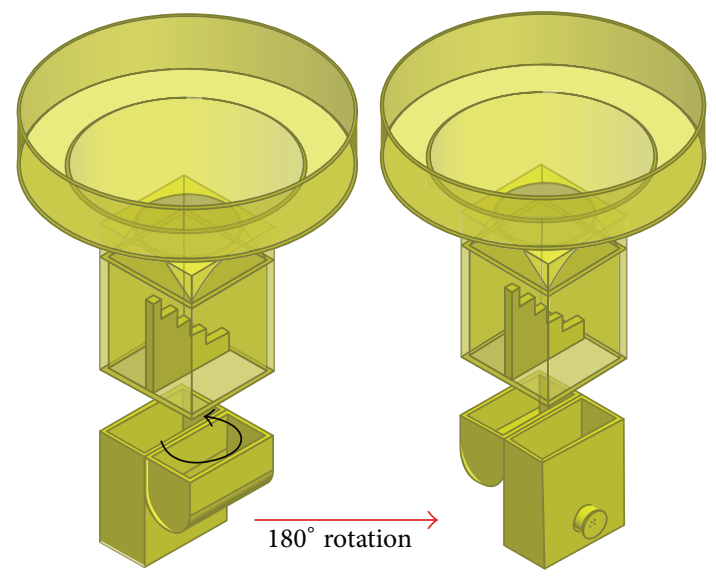

(a)

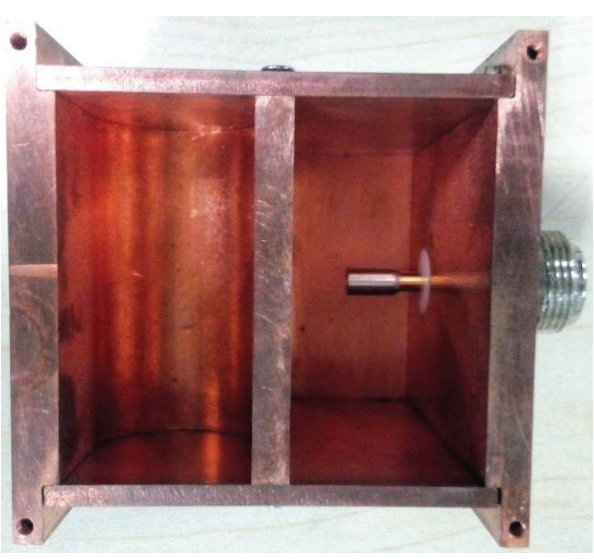

(b)

FIGURE 4: (a) Two connection methods of polarizations; (b) single-feeding system.

Based on the properties outlined previously and parameter scanning, we have arrived at a successful design and have subsequently generalized results; the final dimensions of balance-cavity are tabulated in Table 3 .

2.2. The Similarity of Switchable Polarization. Based on the symmetry properties of structure outlined previously and the equivalence properties of electrical performance, we have arrived at successful results. As Figure 4 illustrated, the coaxial-waveguide converter and the balance-cavity connect with the two input ports of the polarizer in two methods for different polarization modes. The switching can be achieved by the $180^{\circ}$ mechanical rotation of the single-feed system, consisting of a converter and a balance-cavity.

As shown in Figure 5, a good similarity in terms of return-loss, gain, and AR between two kinds of polarization modes is presented, and the simulated cross polarization discrimination of LHCP and RHCP is more than $23 \mathrm{~dB}$.

2.3. The Entire Design Process. The entire design process is mainly divided into three sections: the horn, the polarizer, and the single-feed system. After the successful design and simulation of individual part, integrated simulation is carried out to ensure excellent performance for the final design. Since there is a small degree of interaction between the parts, some adjustments to the dimensions are required during the design. As discussed above, these parameters are a matter of concern while carrying out the integrated simulation. The list of key parameters is as follows: (1) septum width, (2) septum length, (3) balance-cavity length $h_{6}$, (4) balance-cavity, and (5) feed probe. Through defining the objective function and setting key parameters, successful optimization results are achieved within a reasonable computation time. The final dimensions of the antenna after optimization are illustrated in Figure 6 and summarized in Tables 2 and 3.
TABLE 2: Dimensions of the conductive spectrum in Figure 6.

\begin{tabular}{lccccc}
\hline Parameters & $l_{1}$ & $l_{2}$ & $l_{3}$ & $l_{4}$ & $l_{5}$ \\
Values/mm & 8 & 8 & 8 & 8 & 9 \\
\hline Parameters & $w_{1}$ & $w_{2}$ & $w_{3}$ & $w_{4}$ & $w_{5}$ \\
Values/mm & 40.386 & 28 & 20 & 13 & 4 \\
\hline
\end{tabular}

TABLE 3: Dimensions of the proposed antenna in Figure 6.

\begin{tabular}{lcccccc}
\hline Parameters & $h_{1}$ & $h_{2}$ & $h_{3}$ & $h_{4}$ & $h_{5}$ & $h_{6}$ \\
Values/mm & 52 & 51 & 15 & 30 & 25 & 16 \\
\hline Parameters & $h_{7}$ & $h_{8}$ & $h_{9}$ & $r_{1}$ & $r_{2}$ & $r_{3}$ \\
Values/mm & 3 & 1 & 7.5 & 20 & 45 & 64 \\
\hline Parameters & $r_{4}$ & $r_{5}$ & $r_{6}$ & $a$ & $b$ & $t$ \\
Values/mm & 10.1 & 1.57 & 2.14 & 40.386 & 20.193 & 2 \\
\hline
\end{tabular}

\section{Results and Discussions}

The design and simulation of the proposed circularly polarized antenna is accomplished by the commercial microwave simulation software HFSS based on the finite element method. Due to the symmetry of the antenna structure, the property of two polarization modes is almost perfectly in alignment.

The $S$-parameters of the proposed antenna are measured by an Agilent E8363B vector network analyser. Figure 8 shows the simulated and measured $S$-parameters and the discrepancy in resonance frequency may be attributed to the installation procedures and other fabrication accuracies. Due to the symmetry properties of structure outlined previously, good similarity of $S_{11}$ performance between LHCP and RHCP is obtained. It is observed that the measured $\left|S_{11}\right|$ value is below $-10 \mathrm{~dB}$ at $3.88 \mathrm{GHz} 5.12 \mathrm{GHz}$, and the impedance bandwidth is $27.5 \%$. 


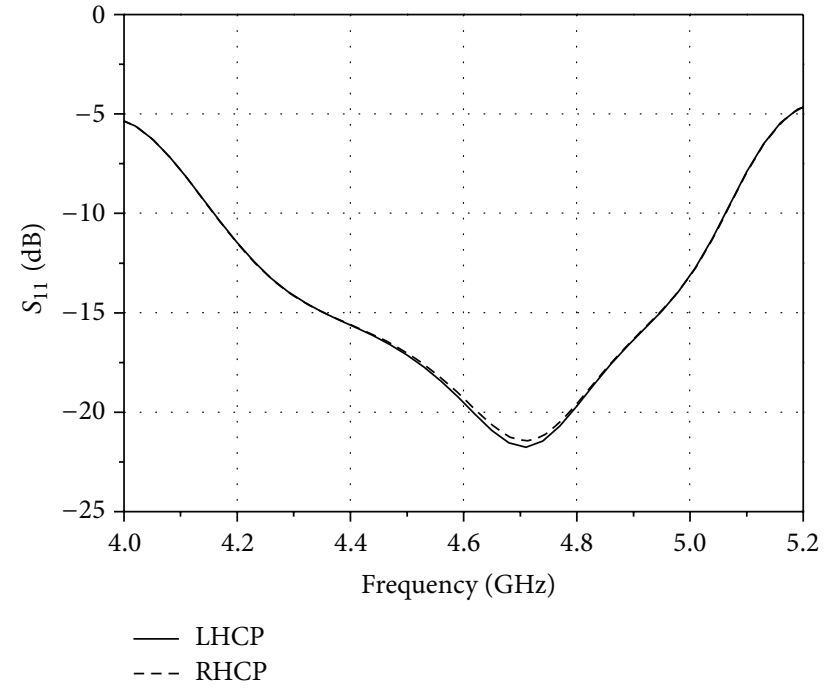

(a)

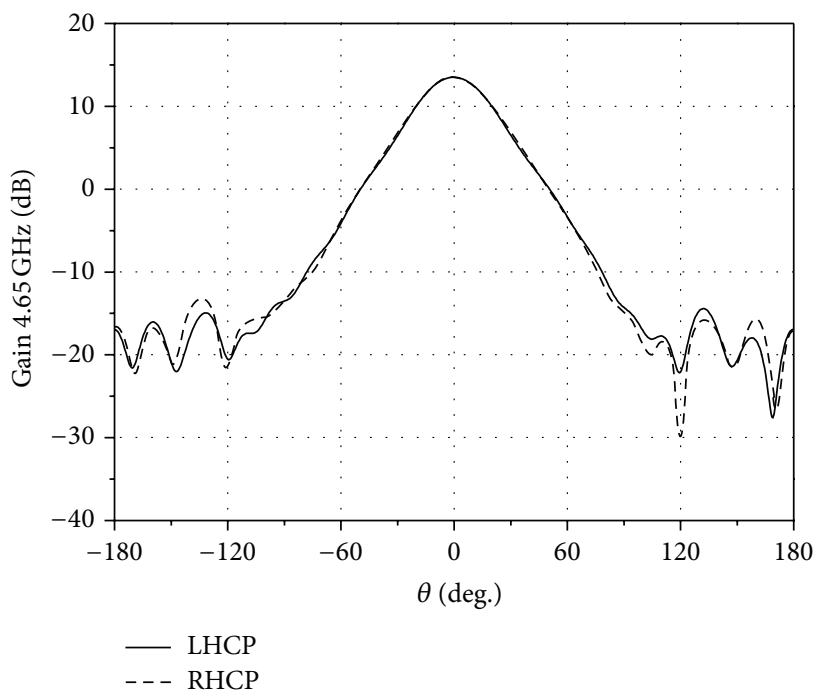

(c)

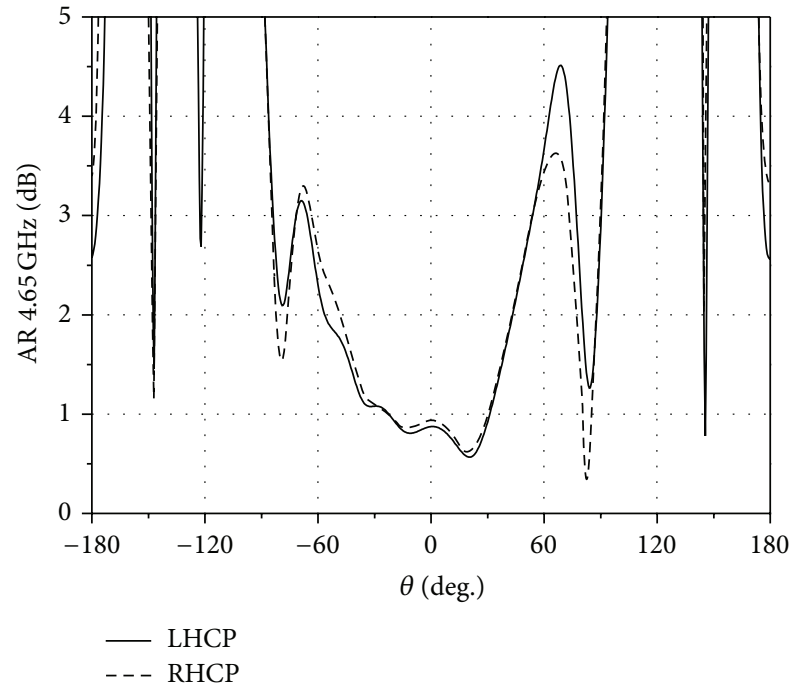

(b)

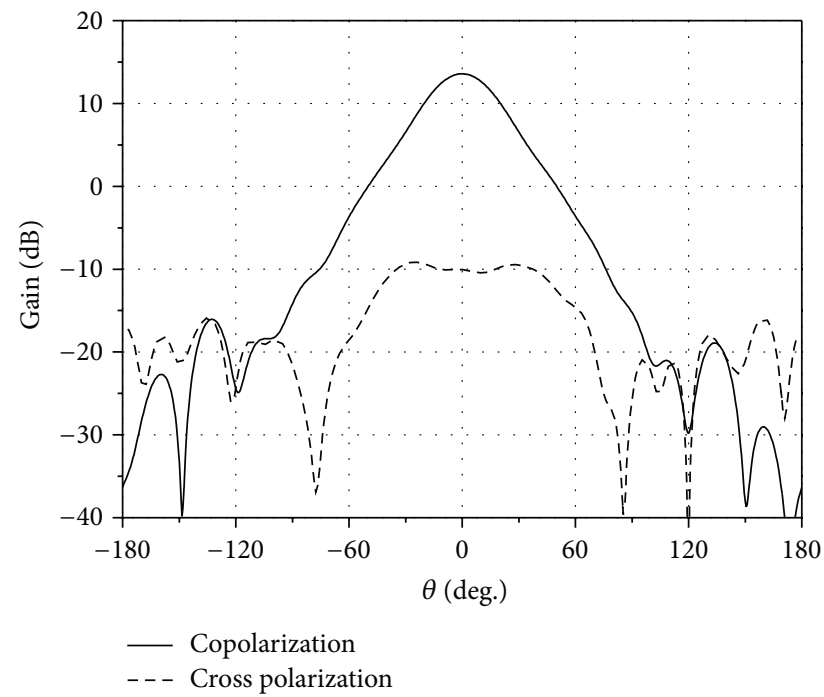

(d)

FIGURE 5: Similarity between LHCP and RHCP.

TABLE 4: The measured result of antenna.

\begin{tabular}{lcccccc}
\hline $\begin{array}{l}\text { Frequency } \\
\text { (GHz) }\end{array}$ & \multicolumn{2}{c}{4.3} & \multicolumn{2}{c}{4.65} & \multicolumn{2}{c}{5} \\
\hline $\begin{array}{l}3 \text { dB-AR } \\
\text { beamwidth }\end{array}$ & $72^{\circ}$ & $76^{\circ}$ & $138^{\circ}$ & $149^{\circ}$ & $116^{\circ}$ & $105^{\circ}$ \\
\hline Gain (dB) & 13.43 & 13.34 & 13.79 & 14.09 & 14.49 & 14.55 \\
\hline
\end{tabular}

As shown in Figure 7, the radiation patterns of the proposed antenna are measured in the far-field anechoic chamber. The measured the simulated LHCP/RHCP radiation patterns and AR-performance at 4.3, 4.65, and $5 \mathrm{GHz}$ are, respectively, plotted in Figures 9 and 10. Good agreement between measured and simulated radiation patterns is achieved. The modular-machining method is used to achieve the final assembly of the proposed antenna due to the complexity of structure, which will bring about some inconsistency between the machined antenna and the HFSS model, thus resulting in some slight discrepancies between the measured and simulated AR results. The antenna exhibits good directional radiation patterns across the entire band and the gains at operation frequencies are about $13.3 \mathrm{~dB}$. It is observed that the LHCP-AR is below $3 \mathrm{~dB}$ when $\theta$ varies from $-39^{\circ}$ to $37^{\circ}$ at $4.3 \mathrm{GHz}$, from $-71^{\circ}$ to $78^{\circ}$ at $4.65 \mathrm{GHz}$, and from $-53^{\circ}$ to $52^{\circ}$ at $5 \mathrm{GHz}$; the RHCP-AR is below $3 \mathrm{~dB}$ when $\theta$ varies from $-34^{\circ}$ to $38^{\circ}$ at $4.3 \mathrm{GHz}$, from $-64^{\circ}$ to $74^{\circ}$ at $4.65 \mathrm{GHz}$, and from $-62^{\circ}$ to $54^{\circ}$ at $5 \mathrm{GHz}$. According to the test data, the $3 \mathrm{~dB}-\mathrm{AR}$ (axial ratio) bandwidth is $18.6 \%$, from $4.23 \mathrm{GHz}$ to $5.1 \mathrm{GHz}$. The gain and $3 \mathrm{~dB}-\mathrm{AR}$ beamwidth of the proposed antenna are tabulated in Table 4. 


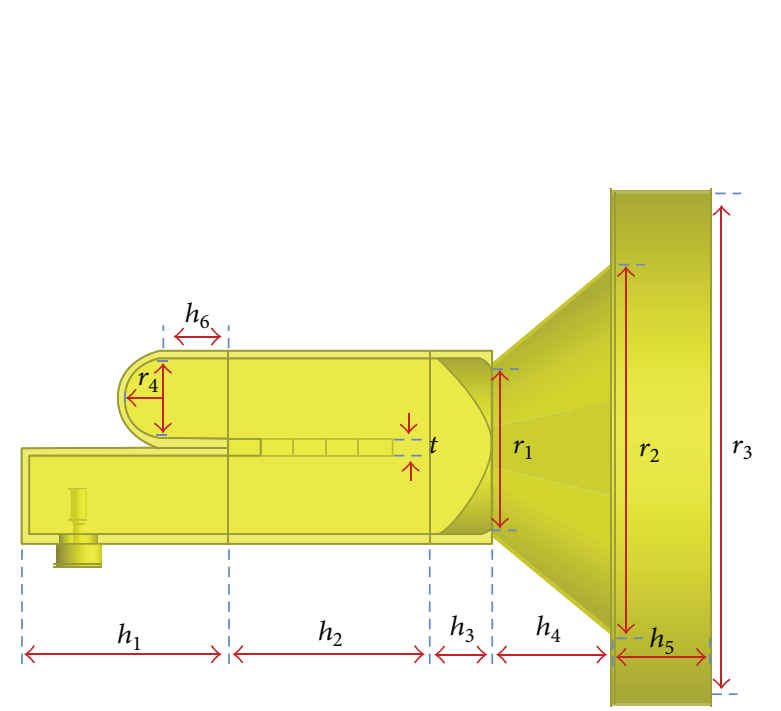

(a)

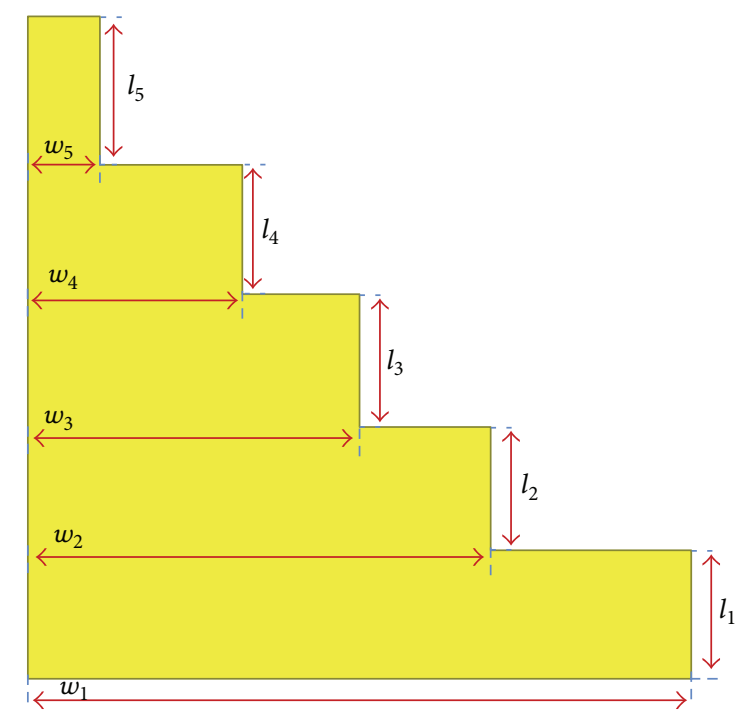

(b)

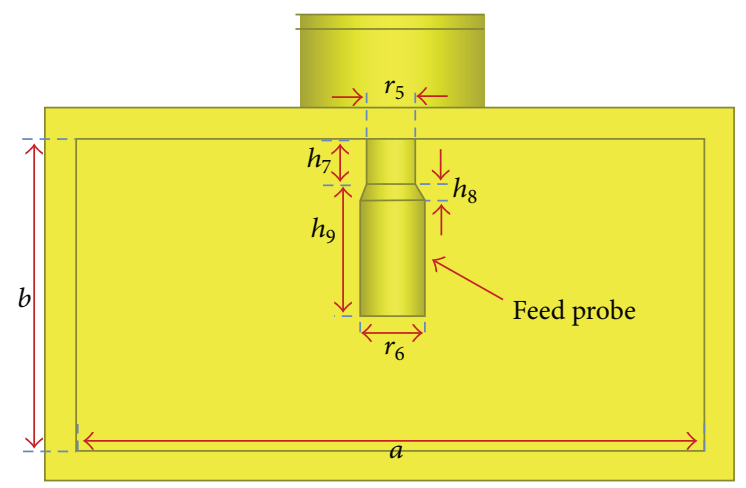

(c)

FiguRE 6: Dimensions of antenna. (a) Right view; (b) conductive septum; (c) coaxial-waveguide converter.

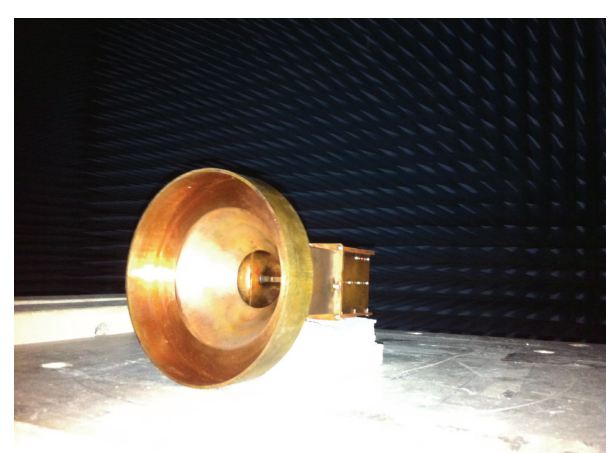

FIGURE 7: Test scenario in far-field anechoic chamber.

\section{Conclusions}

A novel CP horn antenna with switchable polarization by single port feeding is presented, which simplifies the feed structure of antenna and achieves a switch between the lefthand and right-hand circular polarizations. The polarization

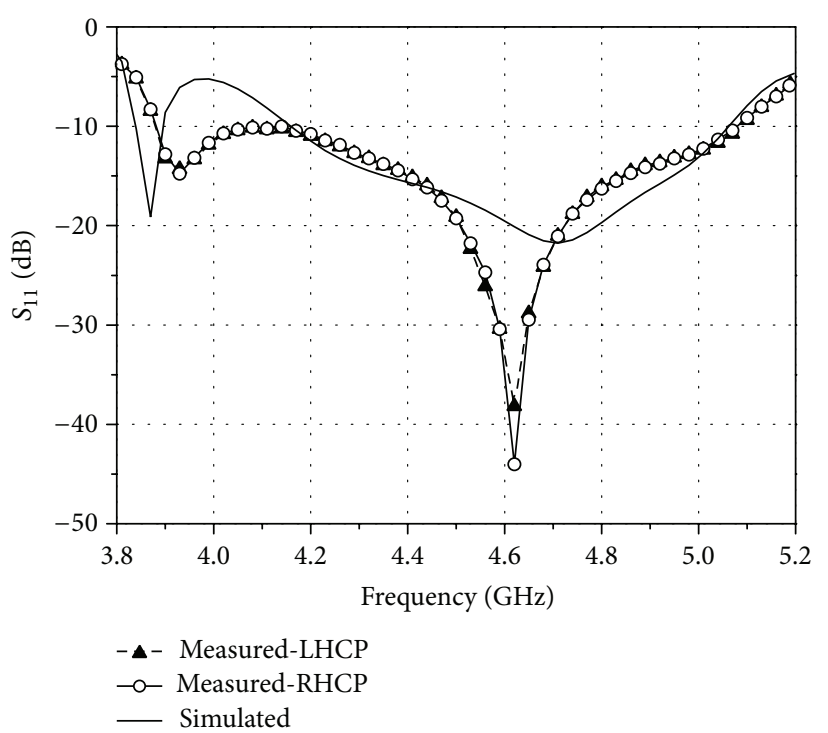

FIGURE 8: Measured and simulated $S$-parameters. 

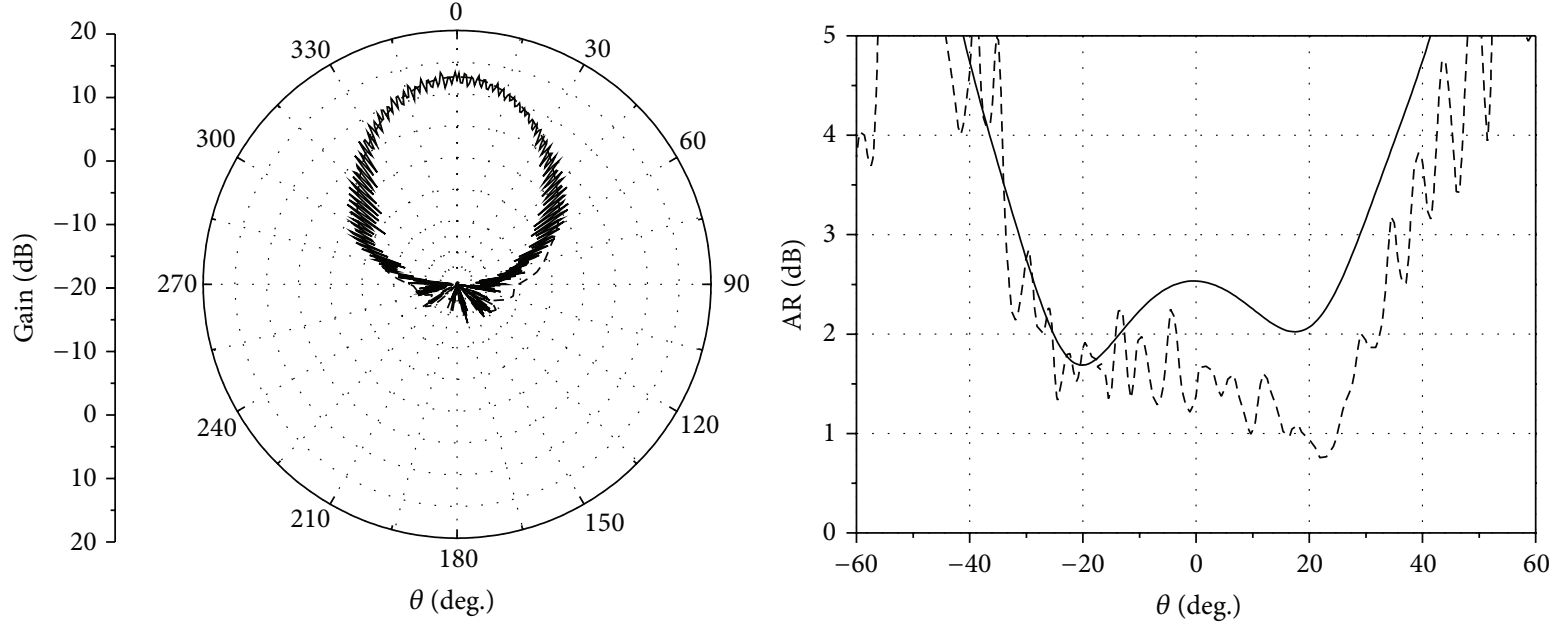

(a)
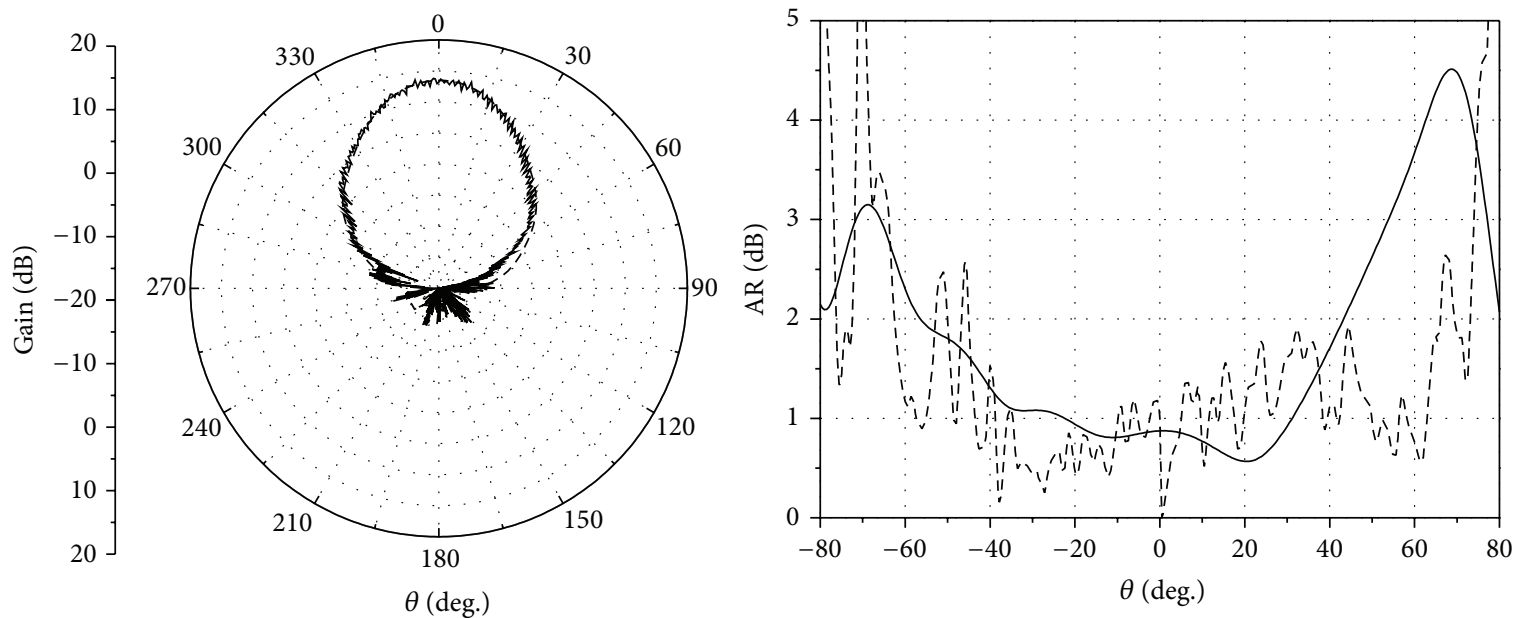

(b)
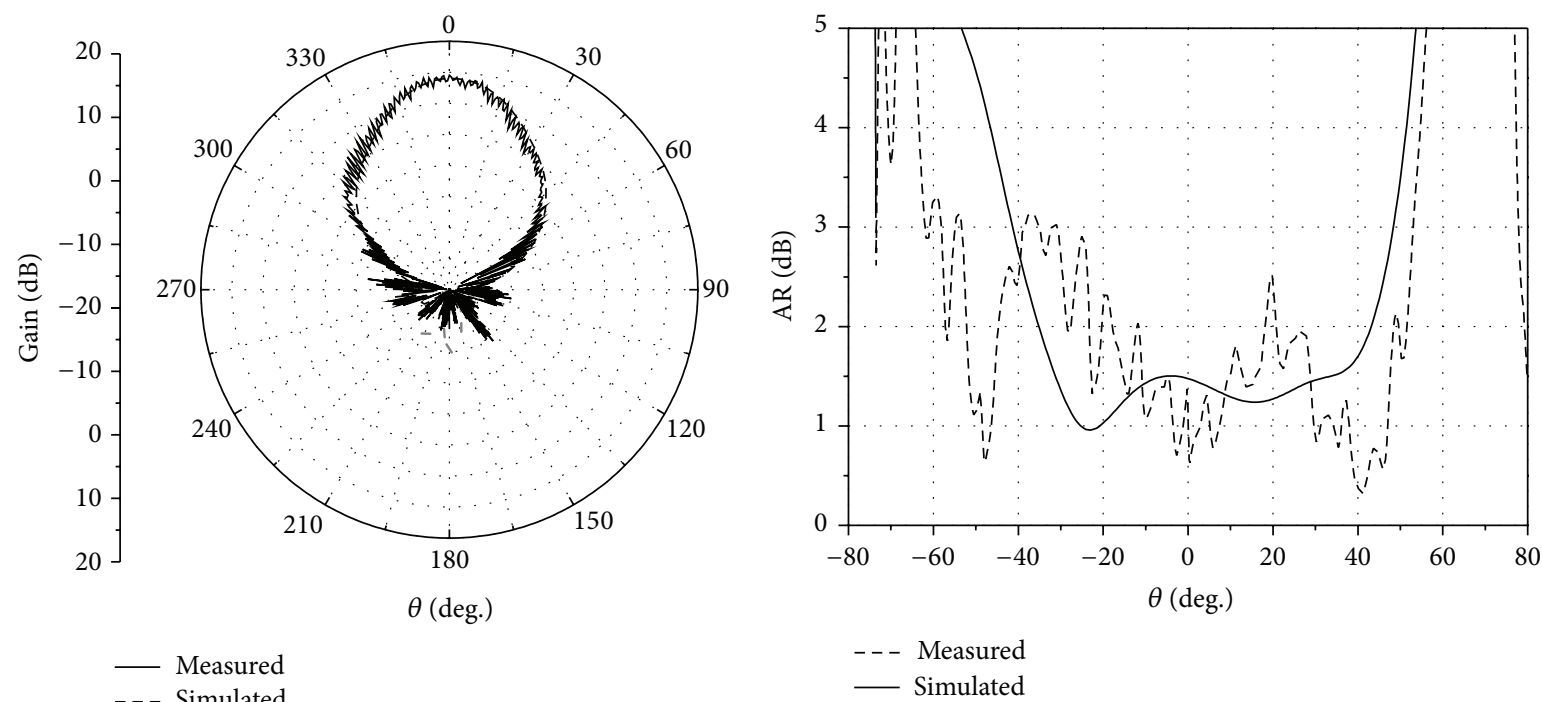

(c)

FIGURE 9: Measured and simulated LHCP radiation patterns and AR. (a) $4.3 \mathrm{GHz}$. (b) $4.65 \mathrm{GHz}$. (c) $5 \mathrm{GHz}$. 

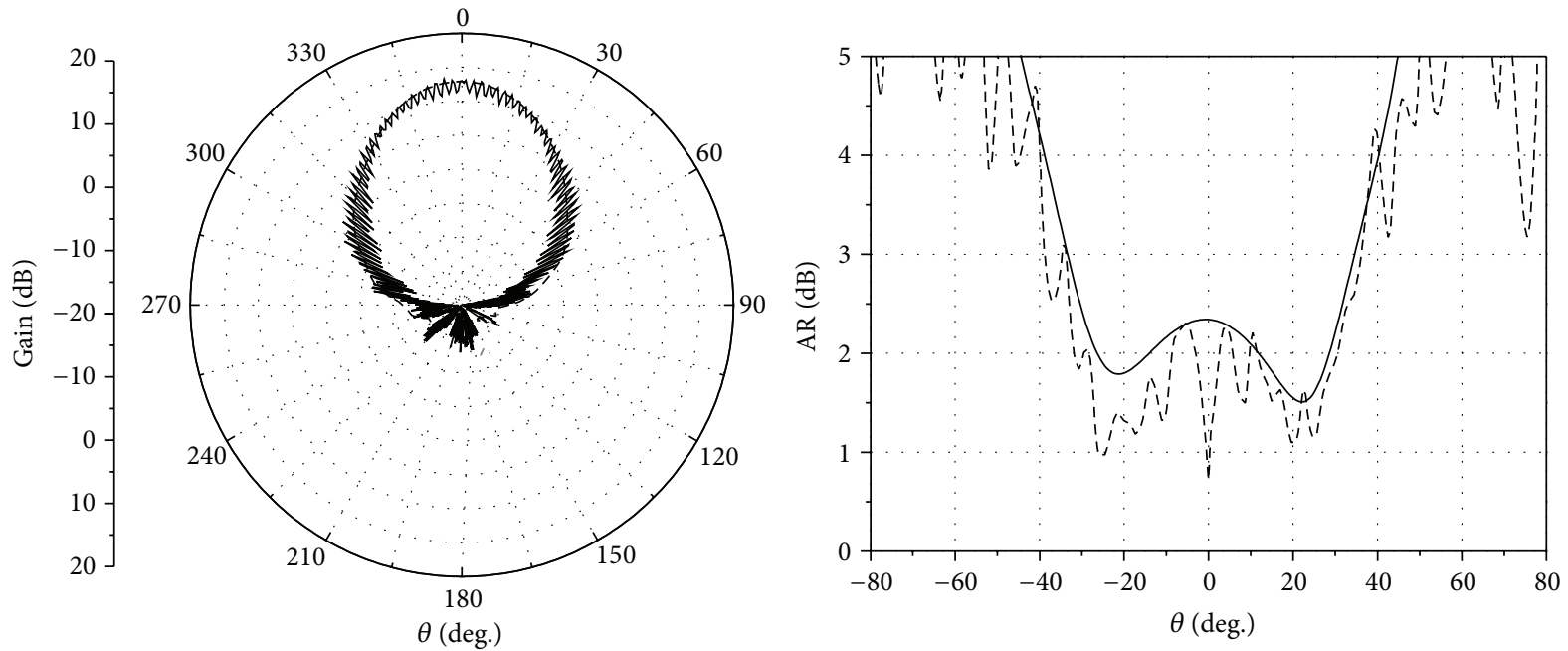

(a)
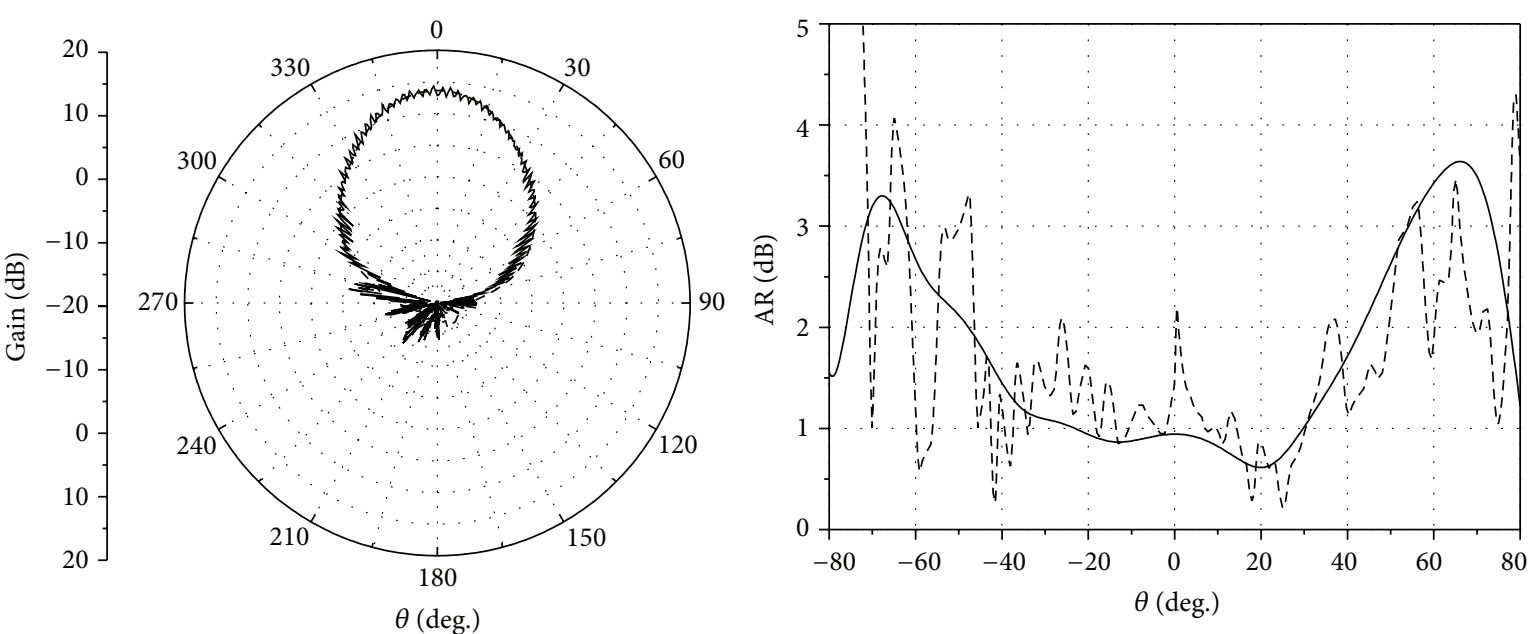

(b)
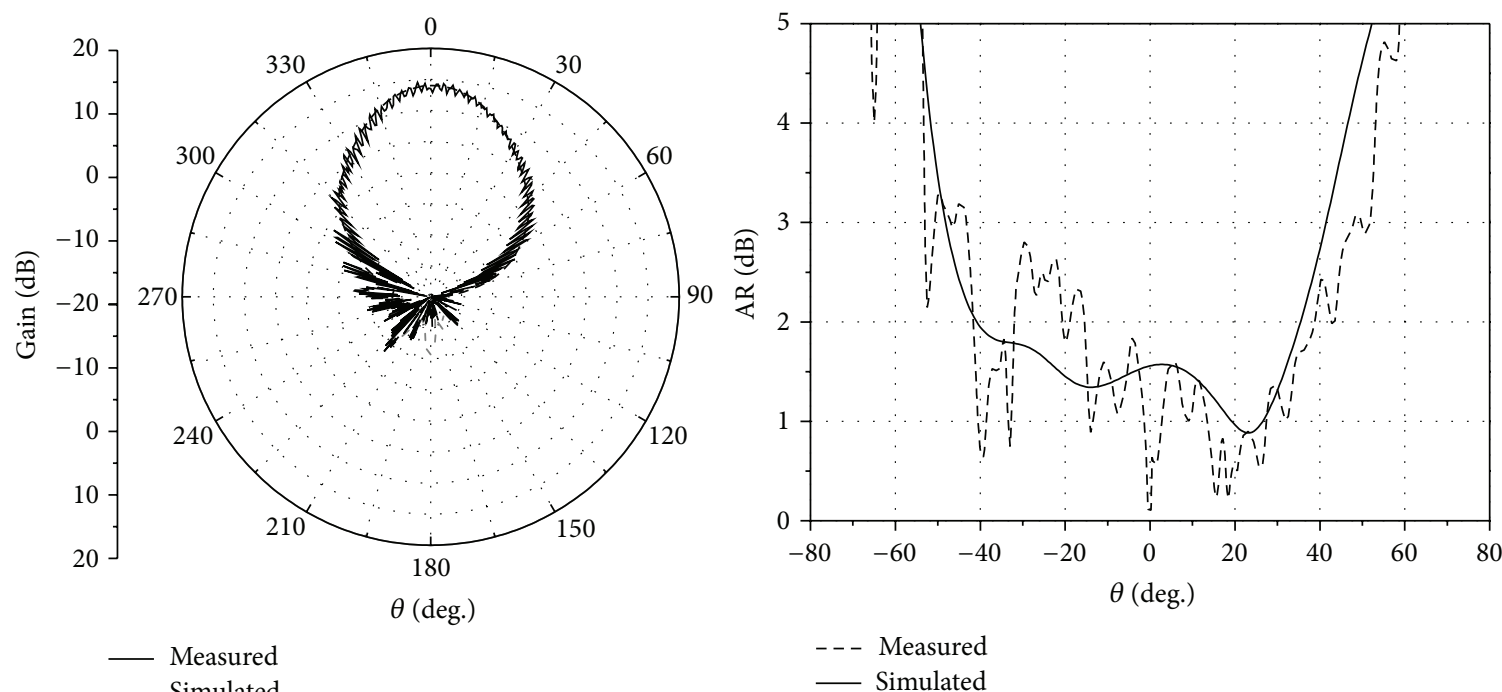

(c)

Figure 10: Measured and simulated RHCP radiation patterns and AR. (a) $4.3 \mathrm{GHz}$. (b) $4.65 \mathrm{GHz}$. (c) $5 \mathrm{GHz}$. 
switch between the left-hand and right-hand circular polarizations can be obtained through changing the connecting position of the coaxial-waveguide converter and the balancecavity. After the measurement, the simulated and measured results show the good consistency. Therefore, it is a promising antenna in communication.

\section{Conflict of Interests}

The authors declare that there is no conflict of interests regarding the publication of this paper.

\section{Acknowledgments}

This work was supported by the National Science and Technology of Major Project (2011ZX03001-007-03), the National Natural Science Foundation (61201058), the Research and Innovation Project of Shanghai Education Commission (12Z112030001), and the Scientific Research Foundation for Returned Overseas Chinese Scholars, State Education Ministry, and the Project of "SMC Excellent Young Faculty."

\section{References}

[1] A. Othman, M. F. Ain, A. A. Sulaiman, and M. A. Othman, "A ka-band horn antenna excited with parasitic dielectric resonator antenna," in Proceedings of the 17th International Conference on Telecommunications (ICT '10), pp. 446-448, IEEE, April 2010.

[2] X. L. Bao and M. J. Ammann, "Monofilar spiral slot antenna for dual-frequency dual-sense circular polarization," IEEE Transactions on Antennas and Propagation, vol. 59, no. 8, pp. 3061-3065, 2011.

[3] J. A. Ruiz-Cruz, M. M. Fahmi, S. A. Fouladi, and R. R. Mansour, "Waveguide antenna feeders with integrated reconfigurable dual circular polarization," IEEE Transactions on Microwave Theory and Techniques, vol. 59, no. 12, pp. 3365-3374, 2011.

[4] T. U. Jang, B. Y. Kim, Y. J. Sung, and Y. S. Kim, "Square patch antenna with switchable polarization using spur-line and PIN diode," in Proceedings of the Asia-Pacific Microwave Conference (APMC '05), vol. 4, IEEE, December 2005.

[5] S.-H. Hsu and K. Chang, "A novel reconfigurable microstrip antenna with switchable circular polarization," IEEE Antennas and Wireless Propagation Letters, vol. 6, pp. 160-162, 2007.

[6] L. Li, Z. Shao, Y. Cheng, Q. Wang, and P. Li, "Design of a novel broadband compact dual polarized horn," in Proceedings of the International Conference on Computational Problem-Solving (ICCP '13), pp. 215-217, IEEE, October 2013.

[7] H. Diez, "High efficiency and compact X band feed for telemetry applications," in Proceedings of the 15th International Symposium on Antenna Technology and Applied Electromagnetics (ANTEM '12), pp. 389-391, June 2012.

[8] J. Bornemann and V. A. Labay, "Ridge waveguide polarizer with finite and stepped-thickness septum," IEEE Transactions on Microwave Theory and Techniques, vol. 43, no. 8, pp. 17821787, 1995.

[9] S. A. Hasan, "Design \& measurement techniques for innovative, high performance, circularly polarized, ultra wideband corrugated horn antenna with septum polarizer for space applications," in Proceedings of the 2nd IEEE International Conference on Microwave Technology and Computational Electromagnetics (ICMTCE '11), pp. 323-326, Beijing, China, May 2011.

[10] J. Esteban and J. M. Rebollar, "Field theory CAD of septum OMT-polarizers," in Proceedings of the IEEE Antennas and Propagation Society International Symposium Held in Conjuction with: URSI Radio Science Meeting and Nuclear EMP Meeting (AP-S '92), vol. 4, pp. 2146-2149, Chicago, Ill, USA, June 1992.

[11] M. J. Franco, "A high-performance dual-mode feed horn for parabolic reflectors with a stepped-septum polarizer in a circular waveguide [Antenna designer's notebook]," IEEE Antennas and Propagation Magazine, vol. 53, no. 3, pp. 142-146, 2011.

[12] M. Chen and G. Tsandoulas, "A wide-band square-waveguide array polarizer," IEEE Transactions on Antennas and Propagation, vol. 21, no. 3, pp. 389-391, 1973. 

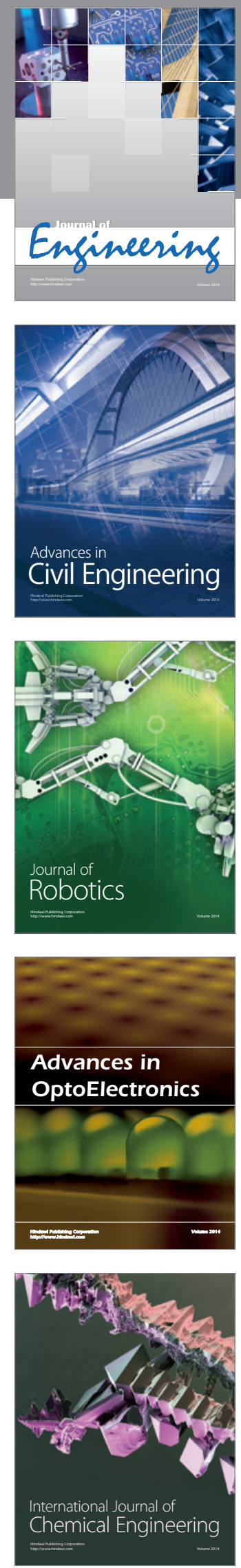

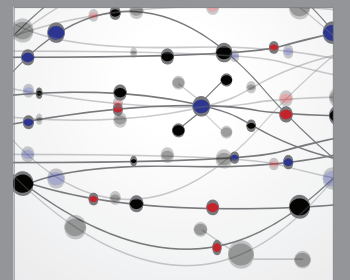

The Scientific World Journal
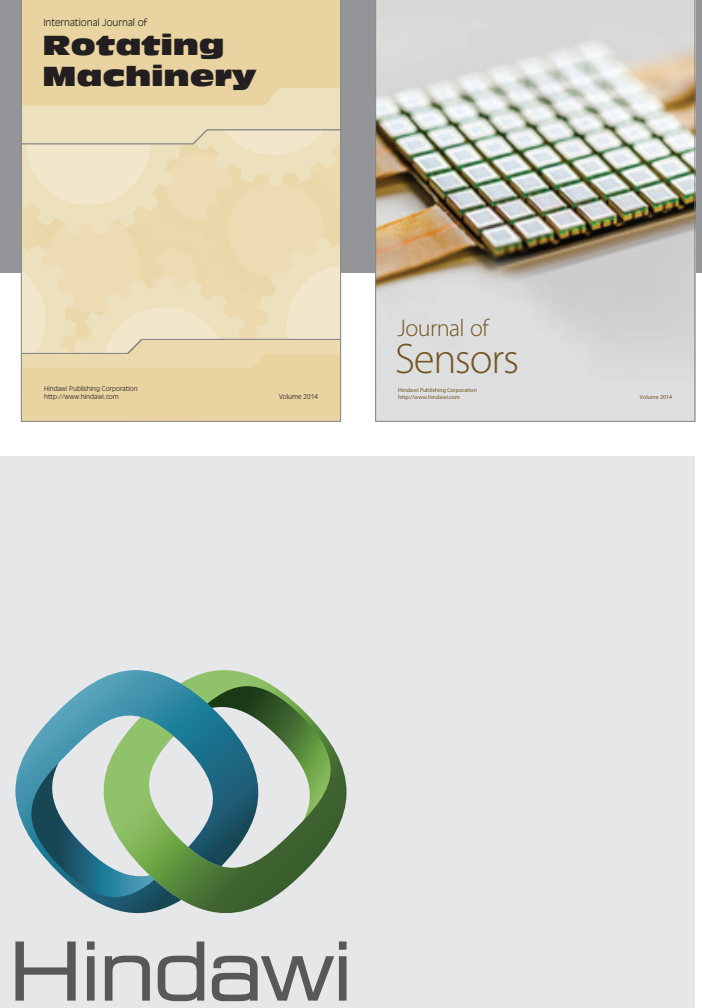

Submit your manuscripts at http://www.hindawi.com
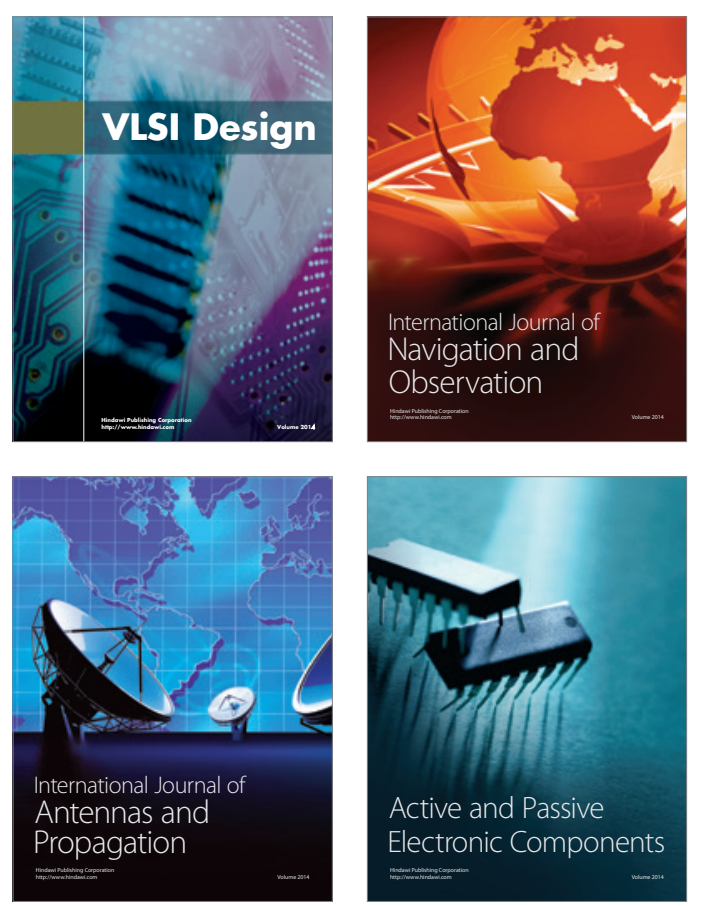
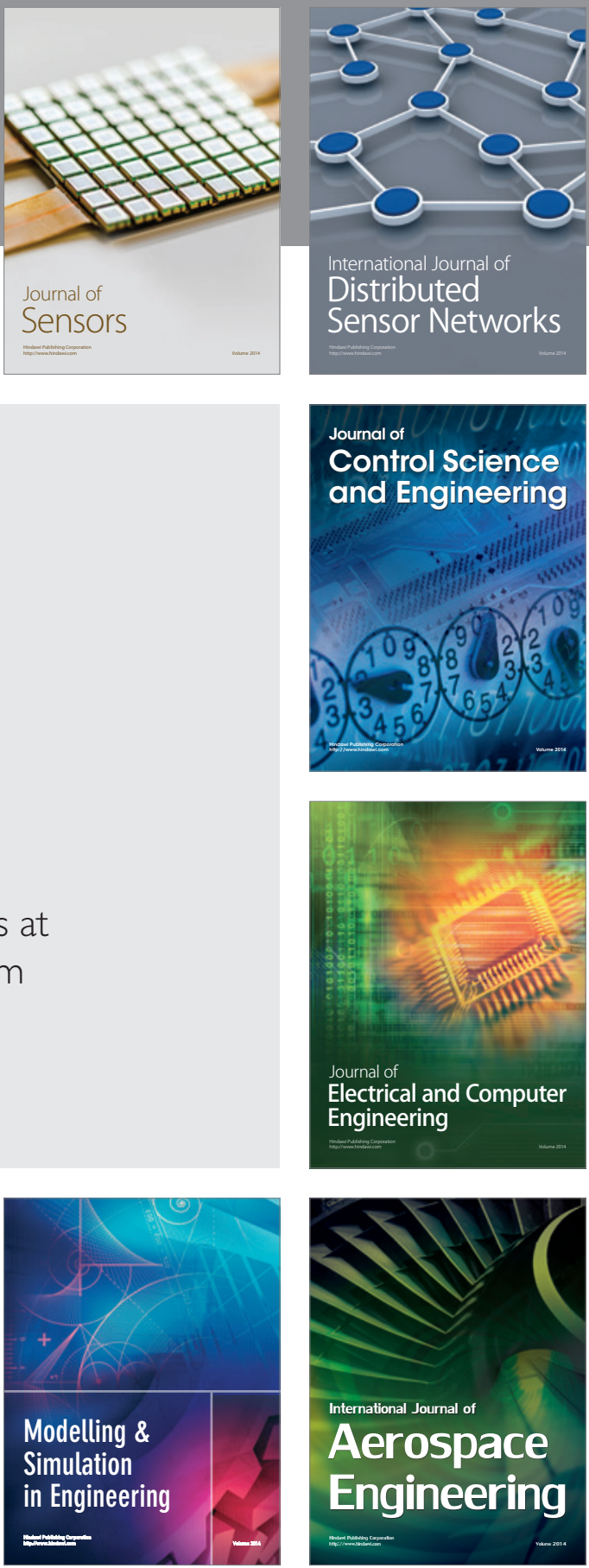

Journal of

Control Science

and Engineering
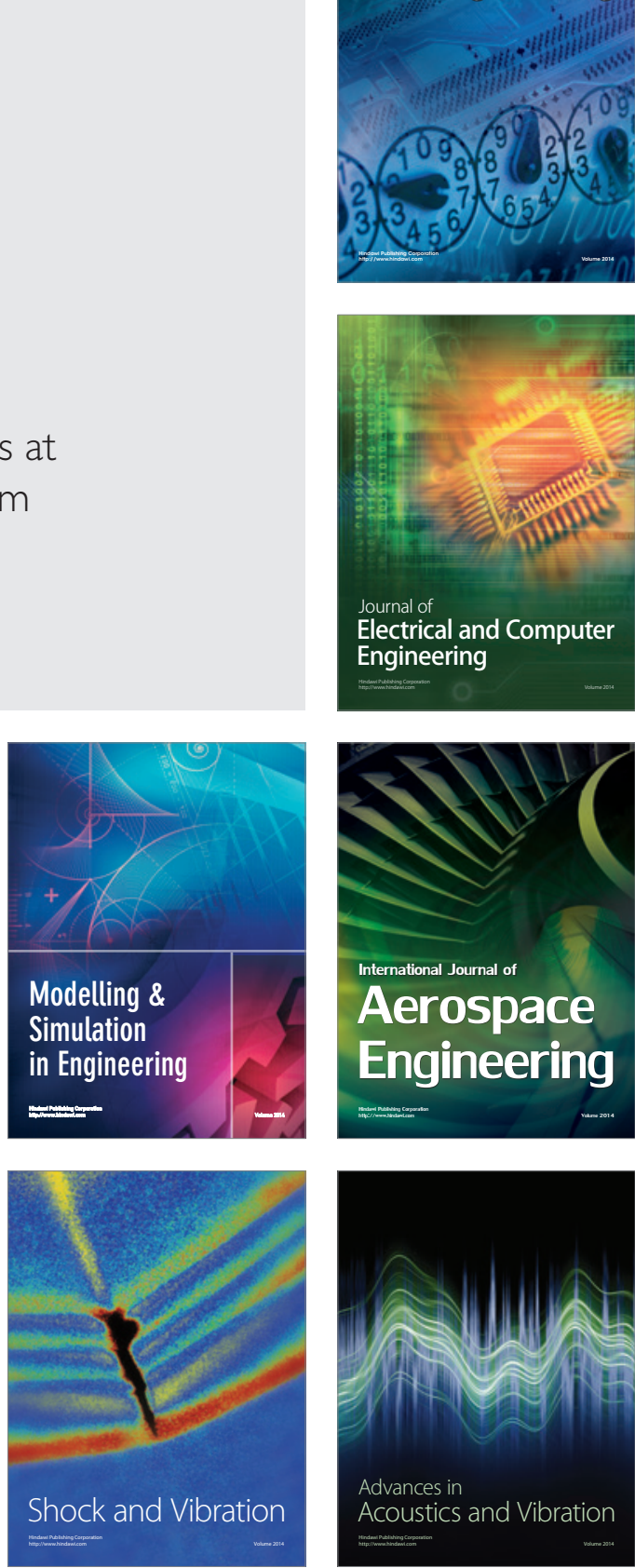